\title{
Cuboid spored Entoloma in Kerala State, India
}

\section{Pradeep $\mathrm{CK}^{1 *}$, Shibu P. Varghese ${ }^{1}$, Vrinda KB ${ }^{1}$ and Baroni $\mathbf{T} \mathbf{J}^{2}$}

${ }^{1}$ Jawaharlal Nehru Tropical Botanic Garden \& Research Institute, Palode, Thiruvananthapuram - 695 562, Kerala State, India

${ }^{2}$ Department of Biological Sciences, State University of New York, College at Cortland, New York, USA - 13045

Pradeep CK, Shibu P. Varghese, Vrinda KB, Baroni TJ 2013 - Cuboid spored Entoloma in Kerala State, India. Mycosphere 4(2), 333-344, Doi 10.5943/mycosphere/4/2/14

Four new cuboid species of Entoloma (E. brunneopapillatum, E. brunneosquamulosum, E. griseolimosum, E. brunneocarnosum) are described, illustrated and discussed based on collections made from Kerala State, India by the authors. A key to the known cuboid species of Entoloma from Kerala State is also provided.

Key words - Entolomataceae - interesting species - new species - quadrate spores - taxonomy

Article Information

Received 5 March 2013

Accepted 9 April 2013

Published online 27 April 2013

*Corresponding author: Pradeep CK - e-mail - drckpradeep@ hotmail.com

\section{Introduction}

During our continuing study on the genus Entoloma of Kerala state, India, four more interesting species with cuboid spores were identified after our first paper on Entoloma went to press (Pradeep et al. 2012). It appears that cuboid spored species of Entoloma are quite well represented in Kerala. Prior to our study, Manimohan et al. (1995, 2002, 2006) described nine new cuboid spored species from the northern part of Kerala. Except for E. haematinum Manim., Leelav. \& Noordel. and E. lomavrithum K.N.A. Raj \& Manim. (Anil Raj \& Manimohan 2012) all of the other species described in those papers have been re-collected by the authors. A key to all the known cuboid species of Entoloma from Kerala is provided together with detailed description of the four new species.

\section{Materials and Methods}

Morphological descriptions are based exclusively on fresh materials. Methods used in the examination of microscopic features are those of Pradeep et al. (2012). Colour notations refer to Kornerup \& Wanscher (1978). Descriptive terms follow Vellinga (1988). All holotypes are deposited at the Herbarium of the Royal Botanic Gardens, Kew (K), and all isotypes and additional materials examined are deposited at the Mycological Herbarium of Tropical Botanic Garden and Research Institute, Trivandrum (TBGT).

\section{Taxonomy}

Entoloma brunneopapillatum C.K. Pradeep \& K.B. Vrinda, sp. nov.

Fig. 1

MycoBank

803535

(http://www.mycobank.org).

Etymology - brunneopapillatum (L.) refers to the brown papillate pileus.

Differing from other small to medium sized mycenoid species with cuboid basidiospores by the glabrous, hygrophanous, translucent-striate, papillate pileus, adnate 
lamellae that lack hymenial cystidia, the cutis or repent hyphae of the pileipellis that contain only plasmatic pigments and the presence of clamp- connections in all tissues.

Basidiomata small to medium, mycenoid. Pileus 10-35 mm diam., conicoconvex, convex to plano-convex with a small subacute to acute papilla often in a shallow depression with age; surface brown (5F5/5F6/6E4/6E5/6F5/6F6) uniformly or slightly darker at the disc, pellucid striate, hygrophanous, glabrous, moist; margin straight, entire to incised. Lamellae adnate, orange white $(5 \mathrm{~A} 2 / 5 \mathrm{~A} 3 / 6 \mathrm{~B} 2)$, up to $7 \mathrm{~mm}$ wide, close with lamellulae of different lengths; edge concolorous, entire. Stipe 30-80 × 2-6 $\mathrm{mm}$, central, cylindric, curved, hollow, brittle, tapering from a slightly enlarged base; surface brown (5D3/5D4/5E4), smooth, glabrous; white mycelial mat rarely observed. Context of the pileus concolorous with the surface, up to 1 $\mathrm{mm}$ thick, soft. Odour none. Spore print pale orange (6A3).

Basidiospores 9-11.5 $\times 7-8 \mu \mathrm{m}$, avL $=$ 10.2, $\mathrm{avW}=7.6, \mathrm{Q}=1.2-1.5$, avQ $=1.34$, quadrate in profile. Basidia 27.5-37.5 $\times$ 9.5$10.5 \mu \mathrm{m}$, clavate, 4 spored. Lamella edge fertile. Cheilocystidia and pleurocystidia absent. Hymenophoral trama regular; hyphae 4.5-15(-32) $\mu \mathrm{m}$ wide, thin-walled, hyaline. Subhymenium pseudoparenchymatous. Pileal trama interwoven; hyphae similar to hymenophoral trama. Pileipellis a cutis of repent hyphae, 5-10 $\mu \mathrm{m}$ wide, interrupted at the disc with suberect hyphal ends with brown intracellular (plasmatic) contents. Stipitipellis a cutis composed of thin-walled hyphae, 4-9.5 $\mu \mathrm{m}$ wide, with pale brown plasmatic contents. Caulocystidia none. Clamp connections and oleiferous hyphae present in all parts.

Habitat - Solitary, scattered or in groups on mud bank among moss or on soil among litter in evergreen forest, July-October.

Material examined - India, Kerala State, Trivandrum District, Palode, JNTBGRI campus, 2 Jul 2012, ShibuTBGT14132; 3 Jul 2012 ShibuTBGT14139 (K (M) 181320 Holotype); 4 Jul 2012 ShibuTBGT14146; 5 Jul 2012 ShibuTBGT14155; 25 Oct 2012 ShibuTBGT14351; $19 \quad$ Oct 2012 ShibuTBGT14334.

\section{Discussion}

Entoloma brunneopapillatum is unique and typical by its small to medium mycenoid basidiomata, brown, papillate, hygrophanous, glabrous pileus, adnate lamellae, brown brittle stipe, quadrate spores, fertile lamellae edge, cuticular pileipellis and presence of clamp connections in all tissues.

Of the known quadrate spored species with a brown pileus and stipe that have been described (Horak 1976, 1977, Hesler 1967, Noordeloos 1981b, 1992, 2004, Pegler 1986, Gates \& Noordeloos 2007, Noordeloos \& Hausknecht 2007, Noordeloos \& Morozova 2010), we compare here those that seem closely or superficially associated with $E$. brunneopapillatum. Entoloma brunneum Petch is one such species that can easily be separated on account of its squamulose pileus and stipe combined with a sterile lamella edge populated by cylindrical to subclavate cheilocystidia. $E$. acutoconicum (Hongo) Horak has a brown to reddish brown pileus covered with vinaceous brown to purple fibrils on the pileus and stipe, making this species distinctive. E. stellatum G. Gates \& Noordel., described from Australia, though apparently close in gross morphology is distinct by its irregularly cruciform smaller basidiospores and heterogenous lamella edge with large lageniform cheilocystidia. $E$. papillatum (Bres.) Dennis is very close to $E$. brunneopapillatum in its gross morphology, however it differs by its large 6-9 angled basidiospores and farinaceous odour. E. spadix Hesler differs by its fibrillose scaly pileus, larger spores and absence of clampconnections. E. gracile Hesler is discernible by its small conic basidiomata, large spores and presence of tufts of caulocystidia. A dark grey brown to reddish brown pileus, twisted striate stipe, cruciform-stellate basidiospores, pileipellis a cutis with a transition to trichoderm and the absence of clampconnections makes E. conferendum (Britz.) Noordel. distinct. E. rhombisporum (Kühn. \& Bours.) Horak is separable from the present species by its yellowish brown pileus and stipe, lageniform to tibiiform cheilocystidia and encrusted hyphae in the pileipellis. Entoloma brunneoquadratum Manim. \& Noordel., E. perinfundibuliforme Manim. \& Noordel., E. 

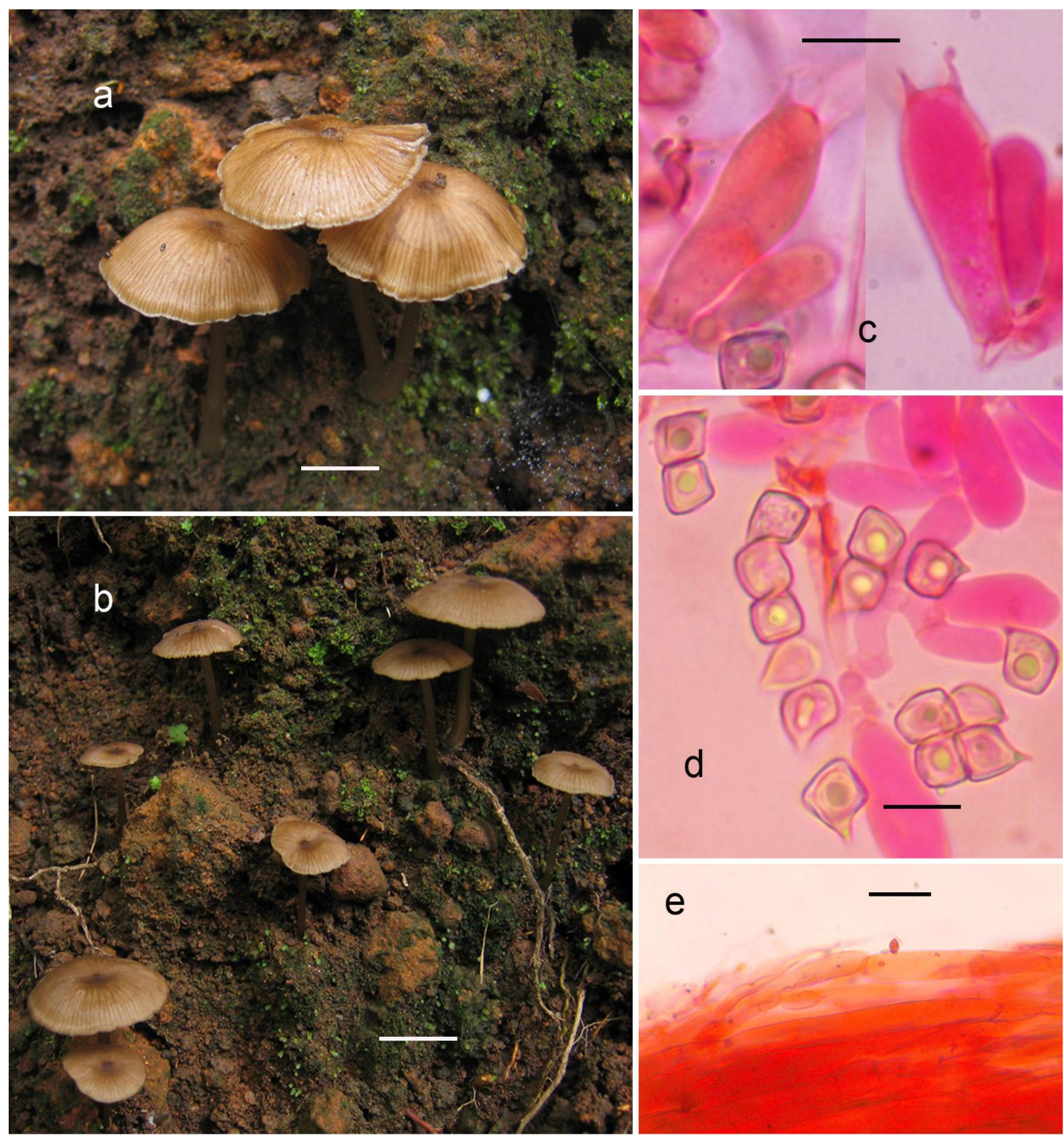

Fig. 1 - Entoloma brunneopapillatum. a, b Habit in situ. c Basidia. d Spores. e Pileipellis.- Bars: a-b $=10 \mathrm{~mm}, \mathrm{c}-\mathrm{d}=10 \mu \mathrm{m}, \mathrm{e}=20 \mu \mathrm{m}$.

keralense Manim. \& Noordel. (Manimohan et al. 2006) and E. lomavrithum KNA Raj \& Manim. (Anil Raj \& Manimohan 2012) are the recently described brown species with quadrate basidiospores from Kerala. The medium sized basidiomata with depressed non-hygrophanous pileus, free lamellae and absence of clampconnections makes E. brunneoquadratum distinct. Large, fleshy basidiomata with deeply depressed pileus, adnexed lamellae with brownish edge and sterile lamella edge with cystidiform elements separates $E$. perinfundibuliforme. Small, delicate, fibrillose squamulose pileus together with cheilocystidia and pleurocystidia makes E. keralense distinct. E. lomavrithum differs by its small brownish omphalinoid basidiomata, tomentose pileus and stipe, and presence of cheilocystidia and clampless hyphae. 
The mycenoid papillate, hygrophanous, smooth pileus coupled with cuboid spores, a cutis construction of the pileipellis with intracellular pigments and hyphae with clampconnections places the present species in subgenus Nolanea (Fr.) Noordel.

\section{Entoloma brunneosquamulosum C.K.} Pradeep \& K.B. Vrinda, sp. nov. Fig. 2, 3 MycoBank 803537 (http://www.mycobank.org).

Etymology - brunneosqumulosum (L.) refers to the brown squamulose pileus.

Distinct from the similar E. brunneum Petch in having larger spores, non-marginate lamellae, hyaline cheilocystidia, presence of clamp connections and exudation of a watery fluid on cutting of the basidiomata.

Basidiomata large, 120-150 mm high, tricholomatoid. Pileus 30-74 mm diam., conic become convex to plano-convex, with a subacute/subobtuse umbo; surface brown (5F8/6D4/6E4), squarrose, appressed to recurved squamulose throughout, denser at the disc, often washed off near margin, nonhygrophanous, dry; margin straight, incised, non striate to striate. Lamellae adnexed, pale yellow/light yellow (3A3/4A4) in buds, orange white (6A2) in mature ones, up to $9 \mathrm{~mm}$ wide, close with lamellulae of different lengths; edge concolorous, smooth, entire. Stipe 65-150 mm $\times$ 3-9 mm, central, cylindric, narrowly hollow, brittle, tapering upwards from a slightly broad base; surface pale yellow (3A3/3D4/4A4) in buds in the lower half become concolorous to the pileus when mature, appressed to recurved squamulose throughout except at the extreme base which is smooth; white mycelial mat present. Context white, up to $2 \mathrm{~mm}$, soft. Producing a colorless watery exudate on cutting the basidiomata. Odour not distinctive or none. Spore print pale orange (6A3).

Basidiospores (12.5-)13-15 $\times(11.5-$ )12-14 (-14.5) $\mu \mathrm{m}$, avL $=14, \mathrm{avW}=13, \mathrm{Q}=$ $1.0-1.2$, avQ $=1.07$, quadrate in profile. Basidia $52.5-68.5 \times 12-16 \mu \mathrm{m}$, clavate, 4 spored, monosporic basidia also occur, rare. Lamella-edge sterile. Cheilocystidia 15-90.5 × 6.5-17.5 $\mu \mathrm{m}$, clavate to cylindroclavate, thinwalled, hyaline. Pleurocystidia none. Hymenophoral trama regular, hyphae 8-16(-
21) $\mu \mathrm{m}$ wide, thin-walled, hyaline. Subhymenium interwoven. Pileal trama interwoven; hyphae similar to hymenophoral trama. Pileipellis an epicutis interrupted at places by tufts of scale cells reaching a trichoderm towards the centre; elements 44.8$144 \times 16.6-29.8 \mu \mathrm{m}$, clavate, cylindric to cylindroclavate, thin-walled with intracellular brown contents. Stipitipellis a cutis, disrupted by scale cells projecting out as cystidioid elements, 32-97.5 × 4-10 $\mu \mathrm{m}$, cylindrical to cylindroclavate, thin-walled with pale brown plasmatic contents. Clamp connections and oleiferous hyphae present in all parts.

Habitat - Solitary, scattered on sandy soil on river banks in evergreen forest, JuneNovember.

Material examined - India, Kerala State, Trivandrum District, Palode, JNTBGRI campus, 29 Jun 2012, PradeepTBGT14123(K (M) 181321 Holotype); 11 Sep 2012, BijeeshTBGT14275; 14 Sep 2012, ShibuTBGT14309; 2 Nov 2012, BijeeshTBGT14409; 5 Nov 2012, BijeeshTBGT14417.

\section{Discussion}

Entoloma brunneosquamulosum is characterized by a distinctive set of features such as medium to large tricholomatoid basidiomata with a nonhygrophanous conic, becoming convex to plano-convex pileus with a subacute/subobtuse umbo, and the surface is covered with appressed to recurved brown squamules; a brown squamulose stipe; lamellae yellowish in young specimens turning pink in mature ones; sterile lamella edge with tufts of hyaline cheilocystidia; presence of clamp connections and the production of a colorless watery exudate on cutting the flesh of the basidiomata.

The present collection is somewhat similar to E. brunneum Petch (Horak 1976, Pegler 1986) but differs markedly because $E$. brunneum produces smaller basidiospores (9$13 \times 8.5-11 \mu \mathrm{m}$ Pegler 1986, 8.5-12 $\mu \mathrm{m}$ Horak 1976), lamellae that are brown marginate, cheilocystidia that are brown pigment filled, lacks clamp connections (Pegler 1986, but Horak (1976) observed clamp connections), and does not produce a watery liquid exudate on cutting of the basidiomata. 

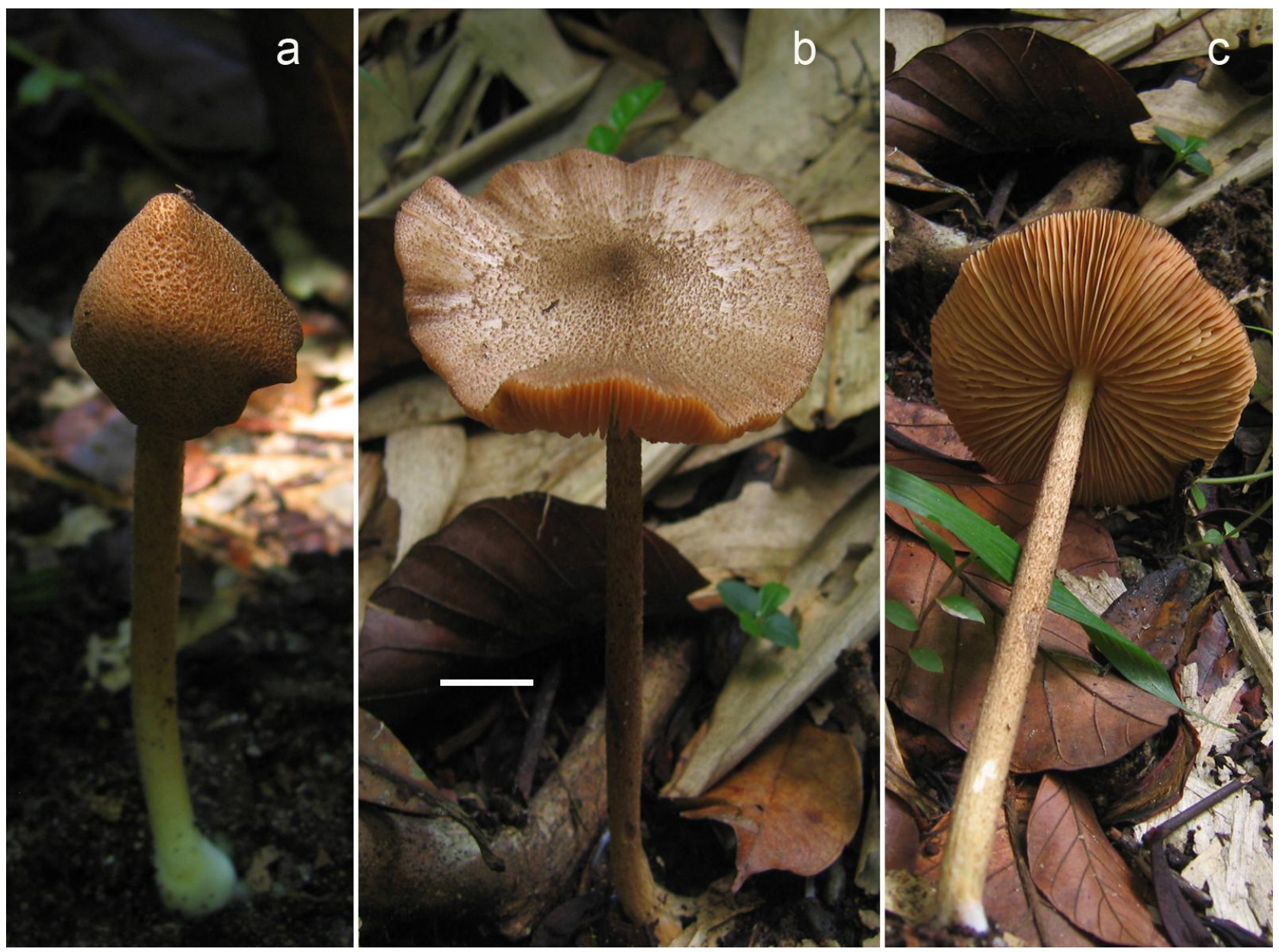

Fig. 2 - Entoloma brunneosquamulosum. a-c Habit in situ - Bar $=10 \mathrm{~mm}$.

Table 1 List of cuboid spored Entoloma from Kerala State, India.

Species

\section{References}

Entoloma albidoquadratum Manim. \& Noordel.

Entoloma aurantioquadratum C.K. Pradeep \& K.B. Vrinda

Entoloma brihadum Manim., Joseph \& Leelav.

Entoloma brunneocarnosum C.K.Pradeep \& K.B.Vrinda Entoloma brunneopapillatum C.K.Pradeep \& K.B.Vrinda Entoloma brunneoquadratum Manim. \& Noordel.

Entoloma brunneosquamulosum C.K.Pradeep \& K.B.Vrinda Entoloma griseolimosum C.K.Pradeep \& K.B.Vrinda

Entoloma haematinum Manim., Leelav. \& Noordel.

\section{Entoloma infundibuliforme Petch}

Entoloma keralense Manim. \& Noordel.

Entoloma lomavrithum K.N.A. Raj \& Manim.

Entoloma perinfundibuliforme Manim. \& Noordel. Entoloma theekshnagandhum Manim., Joseph \& Leelav.

Entoloma vanajum Manim., Joseph \& Leelav.
Manimohan et al. Persoonia 19: 55 (2006)

Pradeep et al. Mycotaxon 120: 338 (2012)

Manimohan et al. Mycological Research 99: 1083 (1995)

This paper

This paper

Manimohan et al. Persoonia 19: 57 (2006)

This paper

This paper

Manimohan et al. Persoonia 17: 625 (2002)

Manimohan et al. Mycological Research 99: 1087 (1995)

Manimohan et al. Persoonia 19: 60 (2006)

Anil Raj KN and Manimohan P. Mycosphere 3: 331 (2012)

Manimohan et al. Persoonia 19: 61 (2006)

Manimohan et al. Mycological Research 99: 1088 (1995)

Manimohan et al. Mycological Research 99: 1087 (1995) 


\section{Key to the cuboid species of Entoloma in Kerala}

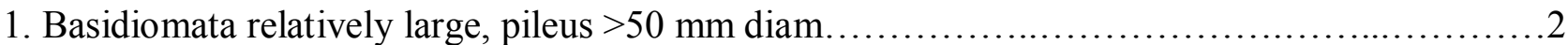

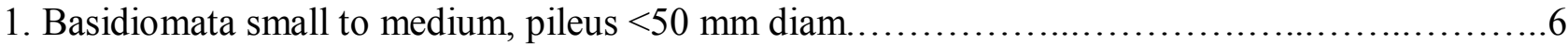

2. Basidiomata pinkish white.

E. brihadum

2. Basidiomata greyish or brownish.

E. perinfundibuliforme

3. Odour distinct, garlic

\section{. perinfundibulforme}

3. Odour none

E. brunneocarnosum

4. Basidiomata clitocyboid, clamp connections absent

4. Basidiomata tricholomatoid, clamp connections present

5. Pileus brown, squamulose, dry, spores large 13-15 × 12-14 $\mu \mathrm{m}$, cheilocystidia present

E. brunneosquamulosum

5. Pileus greyish orange, viscid, spores small 6.5-7.5 $\times 5.5-6 \mu \mathrm{m}$, cheilocystidia absent

E. griseolimosum

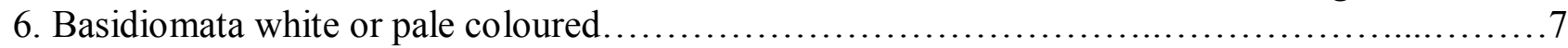

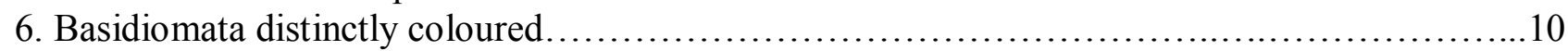

7. Cheilocystidia present, clamp connections present...........................................

7. Cheilocystidia absent, clamp connections absent.....................................

8. Basidiomata discoloring yellowish on bruising; odour strong pungent; spores $8-10 \times 7-10 \mu \mathrm{m} . .$.

.E. theekshnagandhum

8. Basidiomata not discoloring yellowish on bruising; odour none; spores 11-16.5 × 9-14 $\mu \mathrm{m}$. ...

9. Basidiomata clitocyboid; pileus pure white

E. albidoquadratum

9. Basidiomata collybioid; pileus orange white.

E. vanajum

10. Basidiomata very small, thin, pileus $<15 \mathrm{~mm}$ diam...................................... 11

10. Basidiomata small to medium, pileus $>15 \mathrm{~mm}$ diam .................................... 13

11. Basidiomata bright red; cheilocystidia absent.

E. haematinum

11. Basidiomata dark brown, cheilocystidia present ........................................ 12

12. Basidiomata fibrillose squamulose; pleurocystidia and clamp connections present....E. keralense

12. Basidiomata entirely tomentose; pleurocystidia and clamp connections absent....E. lomavrithum

13. Pileus squamulose; cheilocystidia present, pigmented; pileipellis trichoderm.

.E. infundibuliforme

13. Pileus smooth, glabrous; cheilocystidia absent; pileipellis cutis

.14

14. Basidiomata papillate; clamp connections present.

E. brunneopapillatum

14. Basidiomata not papillate; clamp connections absent

E. brunneoquadratum

Entoloma acutoconicum (Hongo) Horak (Horak 1976), originally described from Japan is also somewhat similar in stature but can be separated by its distinct purple-brown fibrils on the pileus and stipe, by its smaller spores (7.5$10 \mu \mathrm{m}$, Horak 1976), by the purple brown lamella edge, by the coloured cheilocystidia and by the presence of pleurocystidia.

E. brunneoquadratum Manim. \& Noordel. described from Kerala (Manimohan et al. 2006), that is better placed in subgenus Nolanea (Fr.) Noordel., differs by its depressed, smooth, glabrous, hygrophanous pileus and stipe, smaller spores, fertile lamella edge and lack of clamp connections in the basidiomata.
The papillate, squamulose pileus, adnexed lamellae, cuboid spores and trichodermial pileipellis with intracellular pigments places E. brunneosquamulosum in the subgenus Inocephalus Noordel. (Noordeloos 1981a).

Entoloma griseolimosum C.K.Pradeep \& K.B.Vrinda, sp. nov.

Fig. 4

MycoBank 803538

(http://www.mycobank.org).

Etymology - griseolimosum (L.) refers to the greyish slimy pileus.

Differes from similar Entoloma by the fleshy tricholomatoid basidiomata, greyish pileus with thick gluten, quadrate spores, fertile lamella edge, gelatinized pileipellis and hyphae 


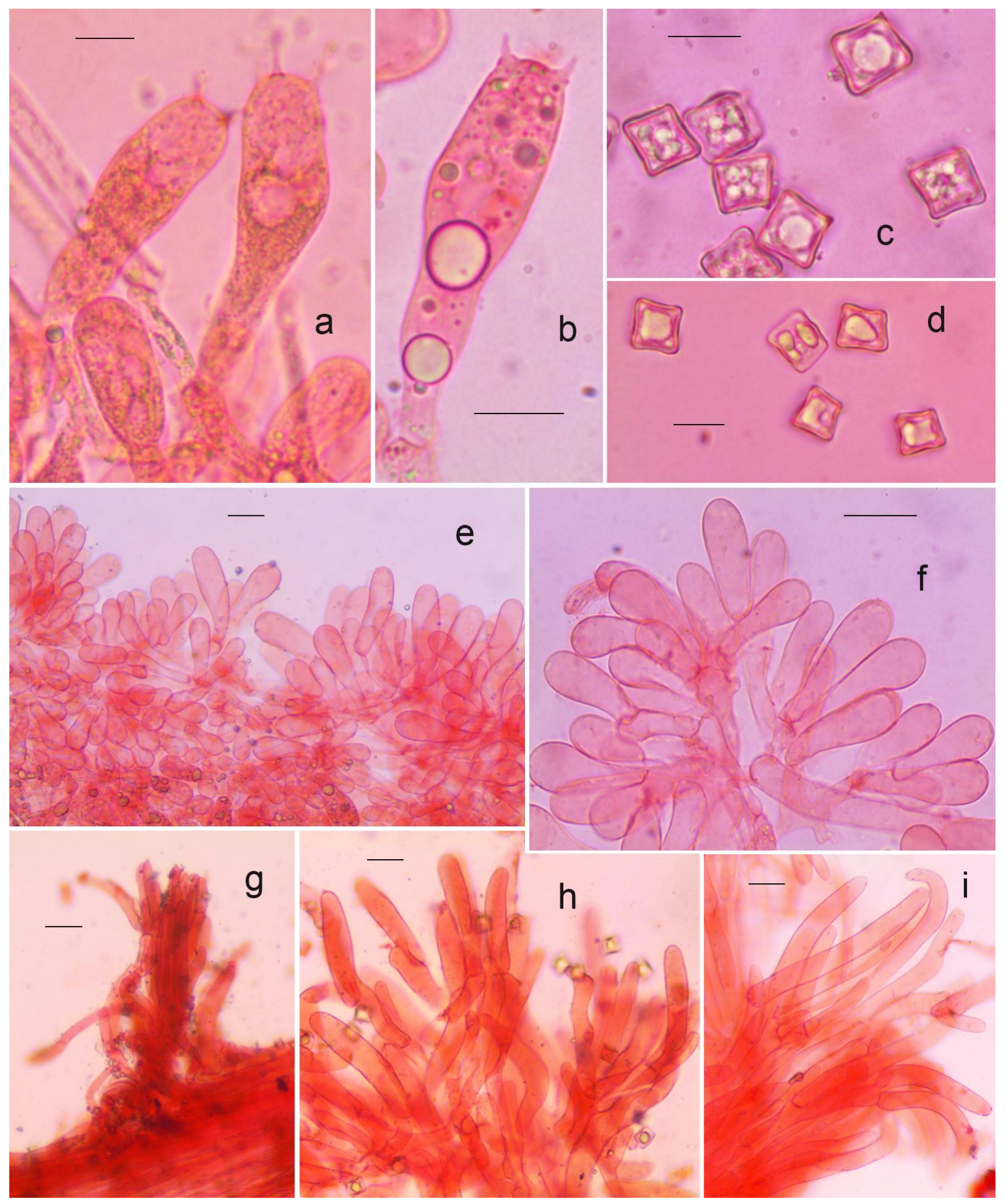

Fig. 3 - Entoloma brunneosquamulosum. a, b Basidia. c, d Spores. e, f Cheilocystidia. g Pileipellis. h pileal elements. $\mathbf{i}$ stipe elements. - Bars: $\mathrm{a}-\mathrm{d}=10 \mu \mathrm{m}, \mathrm{e}-\mathrm{i}=20 \mu \mathrm{m}$.

with clamp connections.

Basidiomata medium to large, fleshy, tricholomatoid. Pileus 32-72 mm diam., convex, broadly-convex, applanate to finally uplifted with a broad obtuse umbo or with a shallow depression at centre; surface greyish orange to clay $(5 \mathrm{~B} 3 / 5 \mathrm{~B} 4 / 5 \mathrm{C} 3 /$
$5 \mathrm{C} 4 / 5 \mathrm{D} 5 / 6 \mathrm{~B} 3 / 6 \mathrm{~B} 4)$ with brownish orange to brown (5C3/5D3/5E5/6F6/6F4/6F5/7F5/7F6) centre/disc, smooth and glabrous, pellucid striate up to half way, strongly hygrophanous, slimy when wet with a gluten cover becoming dry; margin inflexed at first then straight, become uplifted in old ones, entire to incised, 

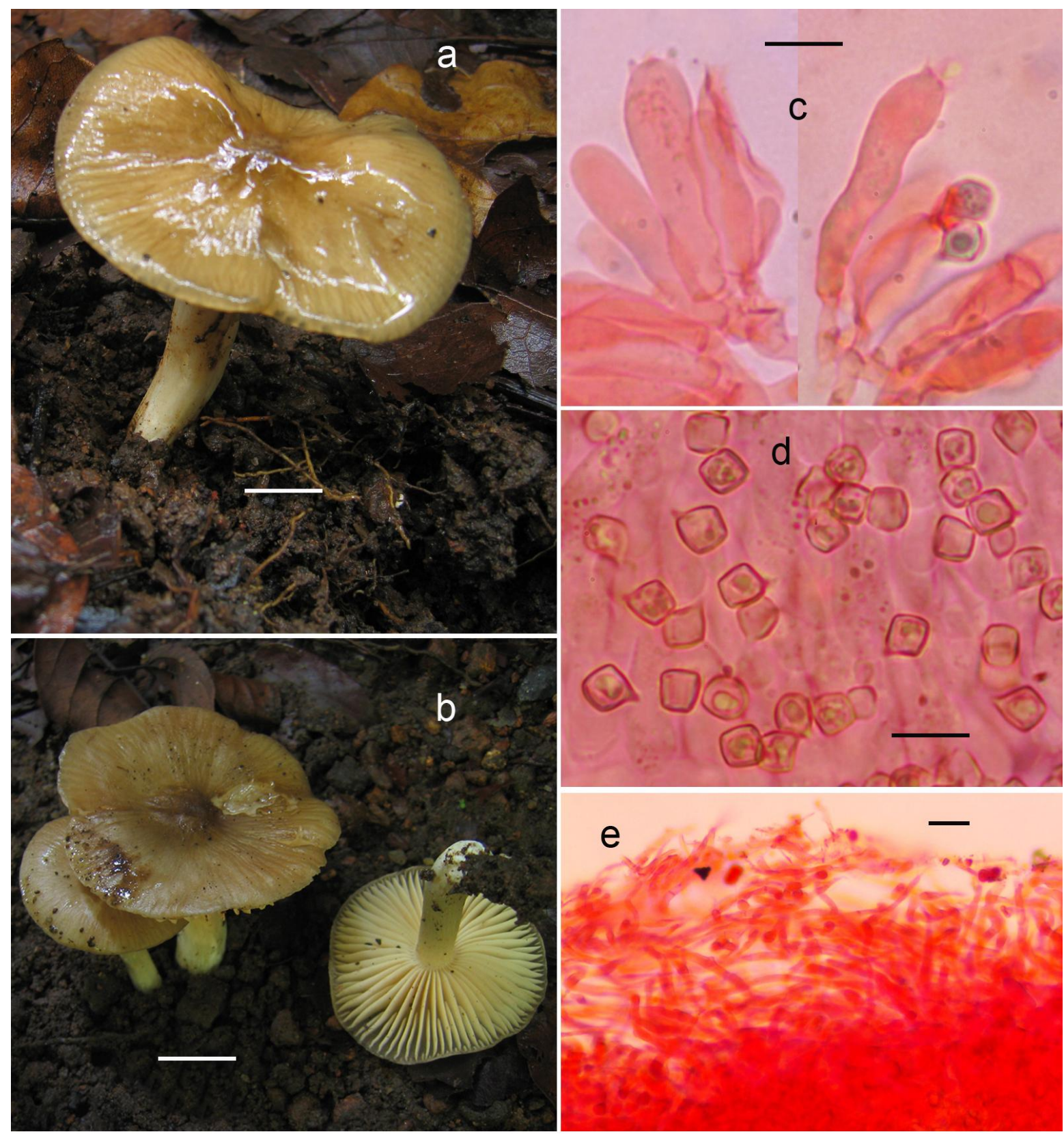

Fig. 4 - Entoloma griseolimosum. a, b Habit in situ. c Basidia. d Spores. e Ixocutis. - Bars: $a-b=$ $10 \mathrm{~mm}, \mathrm{c}-\mathrm{d}=10 \mu \mathrm{m}, \mathrm{e}=20 \mu \mathrm{m}$.

pellucid-striate. Lamellae sinuate adnate, orange white to pale orange $(5 \mathrm{~A} 2 / 5 \mathrm{~A} 3)$ up to $10 \mathrm{~mm}$ wide, close with lamellulae of 1-3 lengths; edge concolorous to the sides, smooth, entire. Stipe 55-105 $\mathrm{mm} \times 5-15 \mathrm{~mm}$, central, cylindric to compressed, solid, equal; surface white, smooth, glabrous, dry; white mycelial Hymenophoral trama regular, hyphae 3-15 (28) $\mu \mathrm{m}$ wide, inflated, thin-walled, hyaline. Subhymenium pseudoparenchymatous. Pileal trama interwoven, hyphae 11-28(-41) $\mu \mathrm{m}$ wide, inflated, thin-walled, hyaline. Pileipellis a gelatinized cutis with hyphae $2-4.5 \mu \mathrm{m}$, thinwalled with pale brown intracellular contents. mat and codons none. Context white, up to 4 $\mathrm{mm}$, soft. Odour none.

Basidiospores 6.5-7.5(-8) $\times 5.5-6 \mu \mathrm{m}$, $\mathrm{avL}=7.17, \mathrm{avW}=5.52, \mathrm{Q}=1.1-1.5, \mathrm{avQ}=$ 1.29 , quadrate in profile. Basidia 34-42.5 $\times 7-$ $8 \mu \mathrm{m}$, clavate, 4 spored. Lamella-edge fertile. Cheilocystidia and pleurocystidia none. Stipitipellis a cutis, hyphae 3-10 $\mu \mathrm{m}$, thinwalled, hyaline. Clamp connections and oleiferous hyphae present in all parts.

Habitat - Solitary, scattered or in pairs on soil among litter in evergreen forest, JuneNovember. 
Material examined - India, Kerala State, Trivandrum District, Palode, JNTBGRI campus, 9 Jun 2009, PradeepTBGT12560; 8 Nov 2011 ShibuTBGT13866 (K (M) 181323 Holotype); 2 Jul 2012 BijeeshTBGT14130

\section{Discussion}

With the following combination of features E. griseolimosum is distinctive species in the Entolomataceae: medium to large fleshy tricholomatoid basidiomata, with a greyish orange, smooth, glabrous, hygrophanous, slimy (gelatinized) pileus, sinuate or adnate lamellae, small cuboid spores, fertile lamella edge, pileipellis a gelatinized cutis and the presence of clamp connections, There are no other taxa in Entoloma that possess this combination of characters. In fact, basidiomata with a slimy pileus surface that result in heavily gelatinized pileipellis hyphae have not been recorded to date (Horak 1980, Noordeloos 2004), although a few taxa are known to produce a thin ixocutis (e.g. E. myrmecophilum (Romag.) Moser and E. lividoalbum (Kühner \& Romagn.) Kubička (Noordeloos 2004). None of the quadrate spored species described by Horak (1976, $1977,1980)$ possess the unique combination of features found in E. griseolimosum.

Entoloma brunneocarnosum C.K.Pradeep \& K.B.Vrinda, sp. nov. Fig. 5 MycoBank 803539 (http://www.mycobank.org).

Etymology - brunneocarnosum refers to the brown fleshy basidiomata

Distinct from $E$. infundibuliformae Petch by hyaline cystidioid elements, large spores and absence of clamp connections.

Basidiomata medium to large, fleshy, clitocyboid. Pileus 50-100 mm diam., convex to uplifted; surface brown (5F8/6D4/5C4/6C3/6E5/6F5), moist to slightly slimy, hygrophanous, silky smooth, glabrous when wet, become fibrillose striate, splitting radially to appear rimose-fibrillose in dry weather; margin inrolled, straight to uplifted, wavy, entire to incised, pellucid striate when wet. Lamellae adnate, flesh (6B3), up to 10 $\mathrm{mm}$ broad, crowded with lamellulae of different lengths; edge concolourous to the sides, entire to eroded. Stipe $50-85 \mathrm{~mm} \times 7-$ $10 \mathrm{~mm}$, central, rarely slightly excentric in some specimens, cylindric to narrowly compressed above, twisted, solid with a slightly broad base, tapering upwards; surface brown (5C3/5E4) fibrillose striate, extreme base white with white mycelial mat. Context white, up to $2 \mathrm{~mm}$, soft. Odour none.

Basidiospores $8-10(-10.5) \times 6.5-8(-$ 8.5) $\mu \mathrm{m}, \mathrm{avL}=9.05, \mathrm{avW}=7.3, \mathrm{Q}=1.05-1.5$, avQ $=1.24$, quadrate in profile. Basidia 32.5$41 \times 9-11 \mu \mathrm{m}$, clavate, 4 spored. Lamella edge sterile with crowded cystidioid elements, tramal in origin, $34.5-70.5 \times 16.5-34.5 \mu \mathrm{m}$, versiform, vesiculose, globose (mostly) with a long narrow stalk, cylindro-clavate, thinwalled, hyaline. Pleurocystidia none. Hymenophoral trama regular; hyphae 4-6.5(12) $\mu \mathrm{m}$ diam., inflated, thin-walled, hyaline. Subhymenium pseudoparenchymatous. Pileal trama interwoven; hyphae similar to hymenophoral trama, thin-walled, hyaline. Pileipellis an epicutis passing to a trichoderm towards the centre, elements, 45-77 $\times 7-12.5$ $\mu \mathrm{m}$, cylindro-clavate, with brown contents; hyphae 3-6.5 $\mu \mathrm{m}$ diam., thin-walled with intracellular brown contents. Stipitipellis a cutis, hyphae 4-7 $\mu \mathrm{m}$, thin-walled, with pale brown intracellular contents occasionally project out in the upper part to form cystidioid elements in some specimens. Clamp connections absent. Oleiferous hyphae present.

Habitat - Solitary, groups or in pairs on forest floor among decayed litter in evergreen tropical forest, June-November.

Material examined - India, Kerala State, Trivandrum District, Palode, JNTBGRI campus, 29 Jul 2002, PradeepTBGT5666; 15 Oct 2003, PradeepTBGT6731; 17 Oct 2003, PradeepTBGT6761; $30 \quad$ Sep 2004, PradeepTBGT8071; 13 Aug 2008, PradeepTBGT11727; 19 Sep 2008, ShibuTBGT11924; 8 Nov 2011, ShibuTBGT13867; 29 Jun 2012, BijeeshTBGT14124; 4 Jul 2012, BijeeshTBGT 14144 (K (M) 181322 Holotype); 5 Jul 2012, BijeeshTBGT14157; 2 Nov 2012, BijeeshTBGT14415.

\section{Discussion}

Entoloma brunneocarnosum is characterized by the following features: clitocyboid basidiomata with deeply depressed, smooth, glabrous, moist to slimy pellucid- 

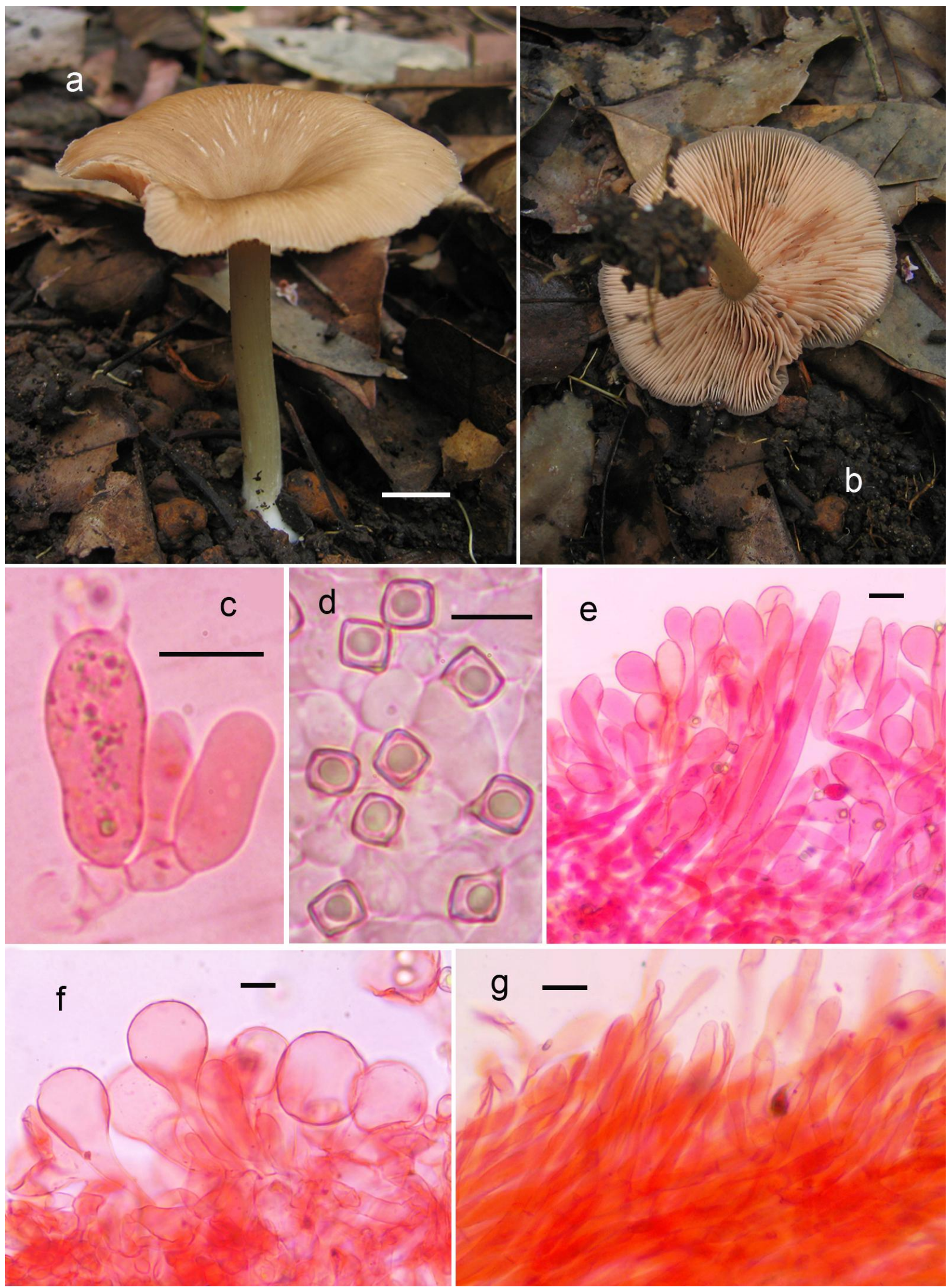

Fig. 5 - Entoloma brunneocarnosum. a, b Habit in situ. c Basidium. d Spores. e, f Cystidia. $\mathbf{g}$ Pileipellis. - Bars: $\mathrm{a}-\mathrm{b}=10 \mathrm{~mm}, \mathrm{c}-\mathrm{d}=10 \mu \mathrm{m}, \mathrm{e}-\mathrm{g}=20 \mu \mathrm{m}$. 
striate pileus which become fibrillose striate to rimose in dry weather; adnate lamellae; concolorous lamella edge; lamella edge sterile with hyaline cystidioid elements which are tramal in origin; pileipellis a slightly gelatinized cutis passing to a trichoderm; clamp connections and odour absent.

According to the macroscopical and microscopical characters, Entoloma brunneocarnosum is a close relative of $E$. infundibuliforme Petch described from Sri Lanka, Malaya, Papua new Guinea (Pegler 1986, Horak 1976). However, squamulose pileus, clamped hyphae, sterile lamella edge with pigmented cheilocystidia and smaller spores separates the latter. $E$. perinfundibuliforme Manim. \& Noordel. described from the northern part of Kerala (Manimohan et al. 2006) is closely related for most of the macro and microscopic characters however, sulcate pileal margin; brown lamella edge; larger spores; presence of clamp connections in all tissues, strong garlic odour and pileipellis a cutis without any gelatinization clearly differentiates $E$. perinfundibuliforme from the present taxon.

The clitocyboid habit, depressed brown pileus, adnate lamellae, cuboid spores and lack of clamp connections indicates its affinity with subgenus Entoloma section Clitopiloides (Romagn.) Noordel. (Noordeloos 1981a).

A list of all cuboid spored Entoloma species from Kerala follows (Table 1), and a key to the species.

\section{Acknowledgements}

The authors are thankful to the peer reviewers for their valuable comments and suggestions. Mr. Jestin, Mr. Bijeesh and Mr. Vishnu are thankfully acknowledged for their help in field exploration.

\section{References}

Anil Raj KN, Manimohan P. 2012 - A new species of Entoloma s.l. associated with earthworm casts. Mycosphere 3, 331334 ,

http://dx.doi.org/10.5943/mycosphere/3/3 16

Gates GM, Noordeloos ME. 2007 Preliminary studies in the genus
Entoloma in Tasmania-I. Persoonia 19, 157-226.

Hesler LR. 1967 - Entoloma in Southeastern North America. Beih. Nova Hedwigia 23, 1-196.

Horak E. 1976 - On cuboid species of Entoloma. Sydowia 28: 171-236.

Horak E. 1977 - Additions to "On cuboidspored species of Entoloma". Sydowia 29, 289-299.

Horak E. 1980 - Entoloma (Agaricales) in Indomalaya and Australasia. Beih. Nova Hedwigia 65, 1-352.

Kornerup A, Wanscher JH. 1978 - Methuen Handbook of Colour. 3ed. Methuen, London.

Manimohan P, Joseph AV, Leelavathy KM. 1995 - The genus Entoloma in Kerala State, India. Mycological Research 99, 1083-1097.

Manimohan P, Leelavathy KM, Noordeloos ME. 2002 - Three new species of Entoloma from Kerala State, India. Persoonia 17, 625-630.

Manimohan P, Noordeloos ME, Dhanya AM. 2006 - Studies on the genus Entoloma (basidiomycetes, Agaricales) in Kerala State, India. Persoonia 19, 45-93.

Noordeloos ME. 1981a - Introduction to the taxonomy of the genus Entoloma sensu lato (Agaricales). Persoonia 11, 121-151.

Noordeloos ME. 1981b - Entoloma subgenera Entoloma and Allocybe in the Netherlands and adjacent regions with a reconnaissance of their remaining taxa in Europe. Persoonia 11, 153-256.

Noordeloos ME. 1992 - Entoloma s.l. Fungi Europaei, vol. 5. Giovanna Biella, Italy.

Noordeloos ME. 2004 - Entoloma s.l. Fungi Europaei, vol. 5a. Edizione Candusso, Italy.

Noordeloos ME, Hausknecht A. 2007 - The genus Entoloma (Basidiomycetes, Agaricales) of the Mascarenes and Seychelles. Fungal Diversity 27, 111-144.

Noordeloos ME, Morozova OV. 2010 - New and noteworthy Entoloma species from the Primorsky Territory, Russian Far East. Mycotaxon 112, 231-255. http://dx.doi.org/10.5248/112.231

Pegler DN. 1986 - Agaric flora of Sri Lanka. Kew Bulletin Additional Series 12, 1- 
MycosphereDoi 10.5943/mycosphere/4/2/14

519.

Pradeep CK, Vrinda KB, Shibu P Varghese, Baroni TJ. 2012 - New species of Entoloma (Basidiomycetes, Agaricales) from Kerala State, India. Mycotaxon 120, 331-342. http://dx.doi.org/10.5248/120.331

Vellinga EC. 1988 - Glossary. In C Bas, Th W Kuyper, ME Noordeloos, Vellinga EC (eds.). Flora Agaricina Neerlandica 1, 54-64. A.A. Balkema, Rotterdam. 